\title{
Outflows from ellipticals: the role of supernovae
}

\author{
Francesca Matteucci and Antonio Pipino \\ Dipartimento di Astronomia, Universitá degli Studi di Trieste, \\ Via G.B. Tiepolo 11, I-34100 Trieste, Italia
}

\begin{abstract}
Models of supernova (SN) driven galactic winds for ellipticals are presented. We assume that ellipticals formed at high redshift and suffered an intense burst of star formation. The role of supernovae of Type II and Type Ia in the chemical enrichment and in triggering galactic winds is studied. In particular, several recipes for SN feed-back together with detailed nucleosynthesis prescriptions are considered. It is shown that SNe of Type II have a dominant role in enriching the interstellar medium of elliptical galaxies whereas Type Ia $\mathrm{SNe}$ dominate the enrichment and the energetics of the intracluster medium.
\end{abstract}

\section{Introduction}

Several mechanisms have been suggested so far for the formation and evolution of elliptical galaxies. One scenario is based on an early monolithic collapse of a gas cloud or early merging of lumps of gas where dissipation plays a fundamental role (Larson 1974; Arimoto \& Yoshii 1987; Matteucci \& Tornambè 1987). In this scenario the star formation stops soon after a galactic wind develops and the galaxy evolves passively since then. Bursts of star formation in merging subsystems made of gas had been also suggested (Tinsley \& Larson 1979); in this picture star formation stops after the last burst and gas is lost via stripping or wind. The alternative and more recent scenario is the so-called hierarchical clustering scenario, where merging of early formed stellar systems in a wide redshift range and preferentially at late epochs, is expected (Kauffmann et al. 1993). The main difference between the monolithic collapse and the hierarchical merging relies in the time of galaxy formation, occurring quite early in the former scenario (at reshifts $z>3$ ) and continuously in the latter scenario. There are arguments either in favour of the monolithic or the hierarchical scenario, but the former one gives a more likely picture since it can reproduce the majority of the properties of stellar populations in ellipticals, in particular some fundamental facts such as that the ellipticals are dominated by old stars (K-giants) and that the $[\alpha / \mathrm{Fe}]>0$ in the dominant stellar population (Worthey et al. 1992; Weiss et al. 1995; Kuntschner et al. 2001). This high $[\alpha / \mathrm{Fe}]$ ratio is the clear signature of the pollution from massive stars. The same occurs in the most metal poor stars in our Galaxy and is due to the fact that SNe II are the main producers of $\alpha$-elements (O, $\mathrm{Ne}, \mathrm{Mg}, \mathrm{Si}$. S and $\mathrm{Ca}$ ) whereas $\mathrm{SNe} \mathrm{Ia}$, which explode with a delay relative to Type II SNe, are thought to be responsible for the production of Fe. Therefore, the high $[\alpha / \mathrm{Fe}]$ ratio in ellipticals argues strongly in favor of 
a short period of star formation during which Type Ia SNe did not have time to substantially pollute the insterstellar medium (ISM).

In this paper we will discuss a monolithic model (Pipino et al. 2002) for the formation and evolution of ellipticals, where these objects suffer a short ( $\leq 1 \mathrm{Gyr}$ ) but intense star formation period halted by a SN driven galactic wind. After the onset of the wind, which devoids the galaxy of all the gas present, star formation is assumed to stop. This is because the galaxy, after the wind, contains hot and rarified gas, a situation which is unfavorable to star formation. The time for the occurrence of the wind, $t_{\mathrm{Gw}}$, is therefore crucial in determining the evolution of the galaxy and the intracluster/intergalactic medium. Therefore, the assumptions about the energy transferred from SNe into the ISM are very important. Unfortunately, very little is known about this feed-back and one has to choose the assumptions which produce a realistic model for ellipticals. In Section 2 we decribe our models; in Section 3 we discuss the condition for the occurrence of a galactic wind and the chemical evolution model; and in Section 4 we present the results and draw a few conclusions.

\section{The model for ellipticals}

The model we are adopting here is described in detail in Pipino et al. (2002). The main feature of the model is that it assumes a strong star formation rate for ellipticals (roughly 20 times stronger than adopted in the solar vicinity) and takes into account in detail the contributions from SN II and SN Ia.

\subsection{SN rates}

It is very important to compute detailed SN rates, taking into account the stellar lifetimes $\left(\tau_{m}\right)$, in order to study the different roles played by SN II and SN Ia in galaxy evolution.

Type II SNe originate from the explosion of massive stars $\left(M>10 \mathrm{M}_{\odot}\right)$, the SN II rate is:

$$
R_{\mathrm{SN} \mathrm{II}}=\int_{M_{\mathrm{m}}}^{M_{\mathrm{M}}} \psi\left(t-\tau_{m}\right) \phi(m) d m
$$

with $M_{m}=10 \mathrm{M}_{\odot}$ and $M_{M}=100 \mathrm{M}_{\odot}$ and $\phi(m)$ being the IMF. Type Ia SNe originate from the thermonuclear explosion of a CO-white dwarf (WD) in a binary system. The binary system can be made of a CO WD plus a red giant star or by two CO WDs. The Type Ia SN rate in the single degenerate case can be written as:

$$
R_{\mathrm{SN} \mathrm{Ia}}=A \int_{M_{\mathrm{Bm}}}^{M_{\mathrm{BM}}} \phi(m) \cdot\left[\int_{\mu_{\min }}^{0.5} f(\mu) \psi\left(t-\tau_{M 2}\right) d \mu\right] d m
$$

where $\mu=\frac{M_{2}}{M_{\mathrm{B}}}$ (with $M_{2}$ mass of the secondary star) and $M_{\mathrm{B}}$, the total mass of the binary system, is defined in the range $3-16 \mathrm{M}_{\odot}$ (see Matteucci \& Greggio 1986).

\subsection{Stellar nucleosynthesis}

Type II SNe produce mainly $\alpha$-elements $(\mathrm{O}, \mathrm{Ne}, \mathrm{Mg}, \mathrm{Si}, \mathrm{S}, \mathrm{Ca})$ and part of Fe. The adopted yields are from Woosley \& Weaver (1995). Type Ia SNe produce mainly Fe-peak elements $\left(\sim 0.6-0.7 \mathrm{M}_{\odot}\right.$ of $\left.\mathrm{Fe}\right)$. The adopted yields are 
from Thielemann et al. (1993) The yields from low and intermediate mass stars $\left(0.8 \leq M / \mathrm{M}_{\odot} \leq 8\right)$ are from Renzini \& Voli (1981).

\section{The development of a galactic wind}

The condition for the occurrence of a wind, where for wind we intend an outflow which carries the gas out of the potential well of the galaxy, is:

$$
\left(E_{\text {th }}\right)_{\mathrm{ISM}} \geq E_{\mathrm{Bgas}}
$$

where $\left(E_{\text {th }}\right)_{\text {ISM }}$ is the thermal energy of the gas and $E_{\mathrm{Bgas}}$ is the potential energy of the gas.

\subsection{The thermal energy of gas}

The thermal energy of gas due to SN and stellar wind heating is:

$$
E_{\mathrm{th}_{\mathrm{ISM}}}=E_{\mathrm{th}_{\mathrm{SN}}}+E_{\mathrm{th}}
$$

with:

$$
E_{\mathrm{th}}=\int_{0}^{t} \epsilon_{\mathrm{SN}} R_{\mathrm{SN}}\left(t^{\prime}\right) d t^{\prime}
$$

and

$$
E_{\mathrm{th}}=\int_{0}^{t} \int_{12}^{100} \varphi(m) \psi\left(t^{\prime}\right) \epsilon_{\mathrm{w}} d m d t^{\prime}
$$

for the contribution from SNe and stellar winds, respectively. The efficiencies of energy transfer from SNe into the ISM is $\epsilon_{\mathrm{SN}}=\eta_{\mathrm{SN}} \epsilon_{\mathrm{o}}$ with $\epsilon_{\mathrm{o}}=10^{51} \mathrm{erg}$ (typical SN energy) and that typical of stellar wind is: $\epsilon_{\mathrm{W}}=\eta_{\mathrm{W}} E_{\mathrm{W}}$ with $E_{\mathrm{W}}=10^{49} \mathrm{erg}$ (typical energy injected by a $20 \mathrm{M}_{\odot}$ star).

The simplest approach is to consider the $\eta_{\mathrm{SN}}$ and $\eta_{\mathrm{w}}$ efficiencies as constant. Bradamante et al. (1998) estimated such efficiencies by computing the ratio between the energy in the shell of the supernova remnant and in the initial blast wave, at the time of the merging of the shell with the ISM, by taking into account results from hydrodynamical calculations. They found that under typical conditions, namely $\epsilon_{\mathrm{o}}=10^{51} \mathrm{erg}, n_{\mathrm{o}}=1 \mathrm{~cm}^{-3}$ (density of the ISM) and $c_{\mathrm{o}}=10^{6} \mathrm{~cm} \mathrm{sec}^{-1}$ (sound speed), $\eta_{\mathrm{SN}}=0.007-0.13$ and $\eta_{\mathrm{W}} \simeq 0.03$. Therefore, the majority of the initial blast wave energy of SNe and stellar winds is radiated away. However, this hypothesis is strictly valid for an isolated object and multiple SN explosions can radically change the situation. In addition, the contributions from different SN types can be different. For example, SNe Ia exploding after Type II should provide more energy into the ISM since they explode in a hot cavity.

In the present model we adopt a more complex formulation which assumes that the $\epsilon_{\mathrm{SN}}$ is varying in time. In particular, we assume the formulation of Cox (1972) for the efficiency of energy injection from SNe:

$$
\epsilon_{\mathrm{SN}}=0.72 \epsilon_{\mathrm{o}} \mathrm{erg}
$$

for $t_{\mathrm{SN}} \leq t_{\mathrm{c}}$, where $t_{\mathrm{c}}$ is the cooling time of a supernova remnant, $\epsilon_{\mathrm{o}}=10^{51} \mathrm{erg}$ is the explosion energy and $t_{\mathrm{SN}}$ is the time elapsed from the $\mathrm{SN}$ explosion. For $t_{\mathrm{SN}}>t_{\mathrm{c}}$ holds:

$$
\epsilon_{\mathrm{SN}}=2.2 \epsilon_{\mathrm{o}}\left(t_{\mathrm{SN}} / t_{\mathrm{c}}\right)^{-0.62} \mathrm{erg}
$$



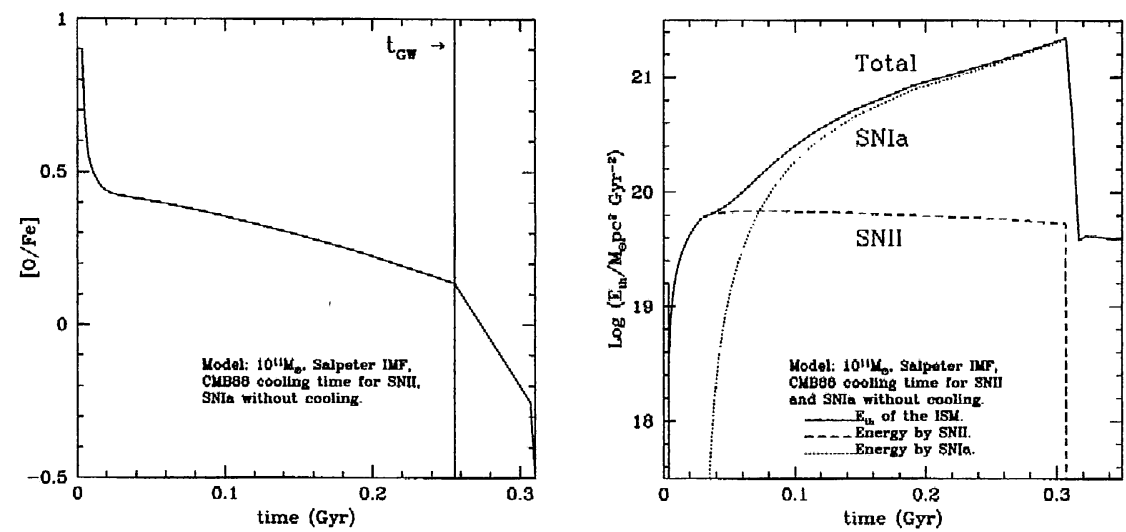

Figure 1. Left: the predicted evolution of the $[\mathrm{O} / \mathrm{Fe}]$ ratio as a function of time in the ISM of an elliptical galaxy. The model assumptions (mass and feedback) are indicated in the figure. In particular, CMB88 refers to Cioffi et al. (1988). The time for the occurrence of the galactic wind is marked. Right: predicted total thermal energy of the gas for the same galaxy. The different contributions from SN II and SN Ia are indicated. SNe Ia are favored energetically since we assumed $\eta_{\mathrm{SN} \mathrm{Ia}}=1$. If $\eta_{\mathrm{SN} \mathrm{II}}=\eta_{\mathrm{SN} \mathrm{Ia}}$ then SN II predominate in the energetics inside the galaxy.

For the cooling time we adopt the formulation of Cioffi et al. (1988) as a function of metallicity:

$$
t_{\mathrm{c}}=1.49 \cdot 10^{4} \epsilon_{\mathrm{o}}^{3 / 14} n_{\mathrm{o}}^{-4 / 7}\left(Z / \mathrm{Z}_{\odot}\right)^{-5 / 14} \mathrm{yr}
$$

It is worth noting that also with these prescriptions $\eta_{\mathrm{SN}}=0.01-0.02$. Recchi et al. (2001) assumed that $\eta_{\mathrm{SN} \mathrm{II}}=0.03$ and $\eta_{\mathrm{SN} \mathrm{Ia}}=1$ in successful chemodynamical models of dwarf irregular galaxies, in order to account for the fact that Type Ia SNe occur with a delay and in an already hot cavity produced by Type II SNe. Pipino et al. (2002) tried several assumptions for SN feedback in ellipticals including this one and concluded that no more than $35-40 \%$ of the initial blast wave energy of SNe II + Ia should heat the ISM in order to have realistic models for ellipticals and the intracluster medium (ICM).

\subsection{The potential energy of the gas}

The total mass of the galaxy is expressed as $M_{\text {tot }}(t)=M_{*}(t)+M_{\text {gas }}(t)+M_{\text {dark }}(t)$ with $M_{L}(t)=M_{*}(t)+M_{\text {gas }}(t)$ being the luminous mass. The binding energy of the gas is computed as in Matteucci (1992 and references therein). For all galaxies here we assumed $M_{\mathrm{dark}} / M_{L}=10$ and $r_{L} / r_{D}=0.1$, being the ratio between the effective radius and the radius of the dark matter core.

\section{Model results}

The galactic winds in the models for ellipticals in the mass range $10^{9-11} \mathrm{M}_{\odot}$ occur on a timescale less than 1 Gyr (Pipino et al. 2002). Therefore, due to the short star formation period assumed for ellipticals, the abundance ratios in their stars show the signature of Type II SN nucleosynthesis, namely $[\alpha / \mathrm{Fe}]>0$, as it is 

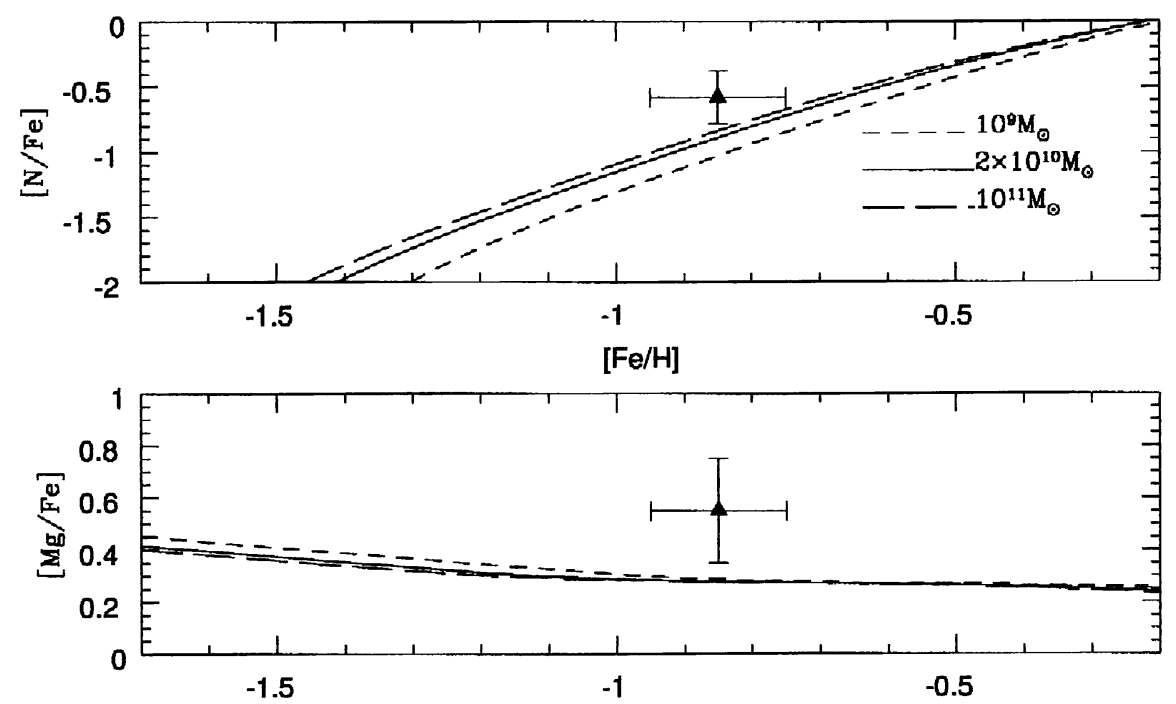

$[\mathrm{Fe} / \mathrm{H}]$

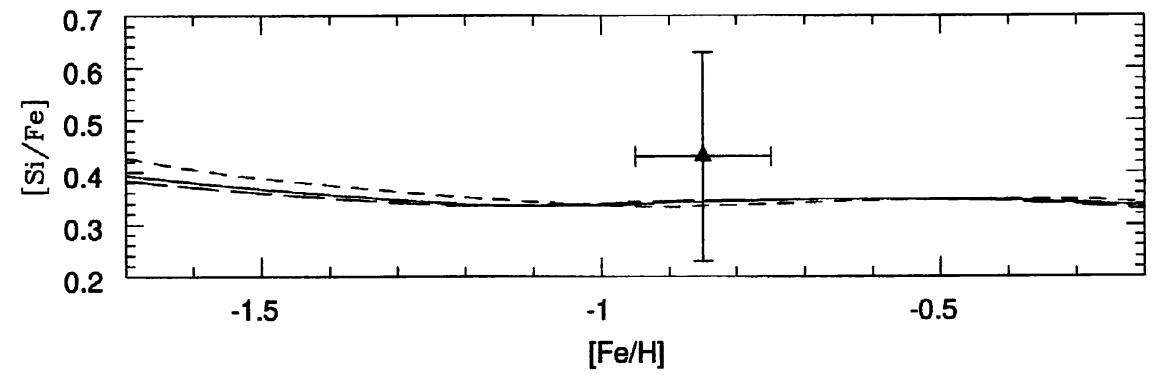

Figure 2. Predicted $[\mathrm{X} / \mathrm{Fe}]$ vs. $[\mathrm{Fe} / \mathrm{H}]$ for models with different initial luminous masses, as indicated in the figure. The data point refers to the Lymanbreak galaxy MS 1512-cB58 from Pettini et al. (2002). The figure is from Matteucci \& Pipino (2002).

evident in figure 1 where we show the predictions of the model for the chemical composition of the gas in a galaxy with initial luminous mass $10^{11} \mathrm{M}_{\odot}$, Salpeter (1955) IMF and feedback prescriptions as in Recchi et al. (2001). In the figure is marked the age for the occurrence of a galactic wind $\left(t_{\mathrm{GW}} \simeq 0.25 \mathrm{Gyr}\right)$. After the wind no more star formation is taking place, so it is evident from the figure that the majority of the stars in this galaxy will show $[\alpha / \mathrm{Fe}]>0$, whereas the gas that goes into the ICM will show $[\alpha / \mathrm{Fe}] \leq 0$. This is due to the occurrence of Type Ia SNe which have a maximum at 0.3-0.5 Gyr (Matteucci \& Recchi 2001), namely after star formation has stopped. The same is true for the energetics of Type II and Ia SNe if we assume $\eta_{\mathrm{SN} I I}=\eta_{\mathrm{SN} \text { Ia }}$. On the other hand, if SNe Ia are assumed to inject all of their initial blast wave energy, the situation is reverted, as shown in Figure 1 (right side); SNe Ia dominate the energetics even before the onset of the wind.This also implies that both the energetics and the chemistry of the ICM are dominated by Type Ia SNe (see Pipino et al. 2002). Finally, 
in Figure 2 we show the predicted $[\mathrm{X} / \mathrm{Fe}]$ vs. $[\mathrm{Fe} / \mathrm{H}]$ relations for ellipticals of different initial luminous mass. Overimposed are the data for a Lyman-break galaxy MS 1512-cB58 at redshift $z=2.7276$ obtained by Pettini et al. (2002).

The good agreement between the predictions for ellipticals and the observations strongly suggest that Lyman-break galaxies at high redshift could be ellipticals in formation, thus supporting the monolithic scenario.

\section{References}

Arimoto, N., Yoshii, Y. 1987, A\&A 173, 23

Bradamante, F., Matteucci, F., D'Ercole, A. 1998, A\&A 337, 338

Cioff, D.F., McKee, C.F., Bertschinger, E. 1998, ApJ 334, 252

Cox, D.P. 1972, ApJ 178, 159

Kauffmann, G., White, S.D.M., Guiderdoni, B. 1993, MNRAS 264, 201

Kuntschner, H., Lucey, J.R., Smith, R.J., Hudson, M.J., Davies, R.L. 2001, MNRAS 323,615

Larson, B.R. 1974, MNRAS 166, 585

Matteucci, F., Greggio, L. 1986, A\&A 154, 279

Matteucci, F., Tornambè, A. 1987, A\&A 185, 51

Matteucci, F. 1992, ApJ 397, 32

Matteucci, F., Recchi, S. 2001, ApJ 558, 351

Matteucci, F., Pipino, A. 2002, ApJ (Letters) 569, L69

Pettini, M., Rix, S.A., Steidel, C.C., Adelberger, K.L., Hunt, M.P., Shapley, A.E. 2002, ApJ 569, 742

Pipino, A., Matteucci, F., Borgani, S., Biviano, A. 2002, New Astron. 7, 227

Recchi, S., Matteucci, F., D'Ercole, A. 2001, MNRAS 322, 800

Renzini, A., Voli, M. 1981, A\&A 94, 175

Salpeter, E.E. 1955, ApJ 121, 161

Thielemann, F.K., Nomoto, K., Hashimoto, M.A. 1993, in: N. Prantzos et al. (eds.), Origin and Evolution of the Elements (Cambridge: CUP), p. 297

Tinsley, B.M., Larson, R.B. 1979, MNRAS 186, 503

Weiss, A., Peletier, R.F., Matteucci, F. 1995, A\&A 296, 73

Woosley, S.E., Weaver, T.A. 1995, ApJS 101, 181

Worthey, G, Faber, S.M., González, J.J. 1992, ApJ 398, 69

\section{Discussion}

ZINNECKER: I have a comment and a question. (i) The comment is that I am surprised that a monolithic origin of ellipticals fits the data better than the hierarchical models. Your result seems to be a warning that not all is well with CDM-based hierarchical galaxy formation. (ii) My question is whether you have also tried IMF slopes different from Salpeter and to what extent you can fit the observed abundance patterns with a flatter/steeper upper IMF?

MATTEUCCI: (i) Concerning hierarchical models: in order to fit the properties of the stellar populations in ellipticals you have to assume an early formation of these objects, clearly at variance with hierarchical models. (ii) Concerning the IMF; we tried Salpeter and flatter IMF (0.95), but we concluded from our results, that the Salpeter IMF works 
better.

DE MELLO: I would like to point out the results from the Sloan Digital Sky Survey ${ }^{1}$ (e.g., Stouhton et al. 2002, AJ 123, 485), suggesting that the local ellipicals have been assembled at earlier epoches (Kauffman, Tremonti, Heckman) than prediced by hierarchical models. I also would like to mention our work (de Mello, Wiklind, Leitherer, these Proceedings) where we show the JCMT-scuBA source SMM 14011. This submm source is the only one with optical identification which is not contaminated by an AGN, it is at $z=2.6$, and it has a SFR of $\sim 1000 \mathrm{M}_{\odot} \mathrm{yr}^{-1}$. The VLT spectrum shows absorbtion lines typical of starbursts, a metallicity $<Z_{\mathrm{LMC}}$, and winds of $\sim 500 \mathrm{~km} \mathrm{~s}^{-1}$. This galaxy fits very well the monolithic scenario.

MATTEUCCI: Thanks for your comment. I can add that our models for massive ellipicals $\left(\sim 10^{12} \mathrm{M}_{\odot}\right.$ of baryonic ions) predict star-formation rates of the order of $1000 \mathrm{M}_{\odot} \mathrm{yr}^{-1}$, in good agreement with the SCUBA data.

OEY: It is very difficult to drive galactic winds from elliptical galaxies, and it is more difficult if the SNe are spatially distributed instead of clustered. Have you considered the different implied escape energetics for TypeIa vs. Type II SNe?

MATTEUCCI: I do not think it is very difficult to produce winds in ellipticals, it depends on the star-formation rate. The predicted star-formation rates of massive ellipticals are up to $1000 \mathrm{M}_{\odot} \mathrm{yr}^{-1}$ and with these rates a wind can develop even if SNe radiate away a large fraction of their initial blast wave energy. Concerning the different spatial distribution of Type I SNe, I do not have a precise answer, since we do not know exactly how to take this into account.

HöfLICH: Type Ia SNe show a wide range in brightness, i.e., the ${ }^{56} \mathrm{Ni}$ production. The subluminous SNe contribute $\sim 20-25 \%$ and the $\mathrm{Fe} / \mathrm{Si}$ ratio is much smaller, Si goes up, etc. Did you include the diversity of SNe Ia?

MATTEUCCI: I did not include the contribution of subluminous Type Ia SNe. However, if they are $20-25 \%$ of the total Type Ia SNe, I do not expect a substantial difference relative to my results.

MARSTON: At high redshifts and low metallicities, stars with masses as high as $300 \mathrm{M}_{\odot}$ have been suggested as being possible. These would also have high energy outputs when they explode. How might such stars affect the results of your models?

MATTEUCCI: It depends on how many such objects are likely to be present. If there is a substantial fraction of them (depends also on the IMF), then the evolution of ellipticals would be even faster than I have shown. However, only one generation of massive stars is likely to produce a negligible effect on chemical evolution.

Noмото: If you assume that Type Ia SNe start exploding $0.5 \mathrm{Gyr}$ after the starburst, would it make a difference in your results?

MATTEUCCI: Yes, it would probably delay the onset of galactic winds, but I expect no substantial differences in the predicted abundance patterns in stars in ellipticals. On the other hand, in the solar vicinity this would produce a longer plateau than observed for the $\alpha$-elements relative to Fe.

${ }^{1}$ http://www.sdss.org 